\title{
Image Processing Based Production Flaw Detection in Knitting
}

\author{
J. Ramprabu, Aswini N
}

\begin{abstract}
One of the major problems in production is that the number of faults occurred while producing a fabric, since it directly influences productivity. To overcome the losses, this paper provides a system with special surveillance to detect the knitting process, identify and locate faults during manufacturing, by inspecting the fabric. The system also gives the user a valuable set of data related with production. This examination has demonstrated that picture handling can possibly give solid estimations to recognizing abandons in sewed textures. On-line texture deformity location was tried consequently by contrasting texture pictures caught by a computerized camera. In this way, it is demonstrated that the created picture catching and investigation framework is equipped for halting the round sewing machine by utilizing processor when a deformity is caught by the web camera.
\end{abstract}

Index Terms-Image processing, Circular knitting machine, Web camera.

\section{INTRODUCTION}

Any variety in the weaving procedure of the texture needs to examine and address. Imperfections fall into this classification. When they show up, redress is required; this is tedious and some of the time brings about the texture's dismissal. Texture imperfection location has been a need in the sewing business. Studies demonstrate that off base assessment of textures has prompted texture imperfections being missed, which consequently has viably influenced the quality and following expenses of the texture and article of clothing fabricating.

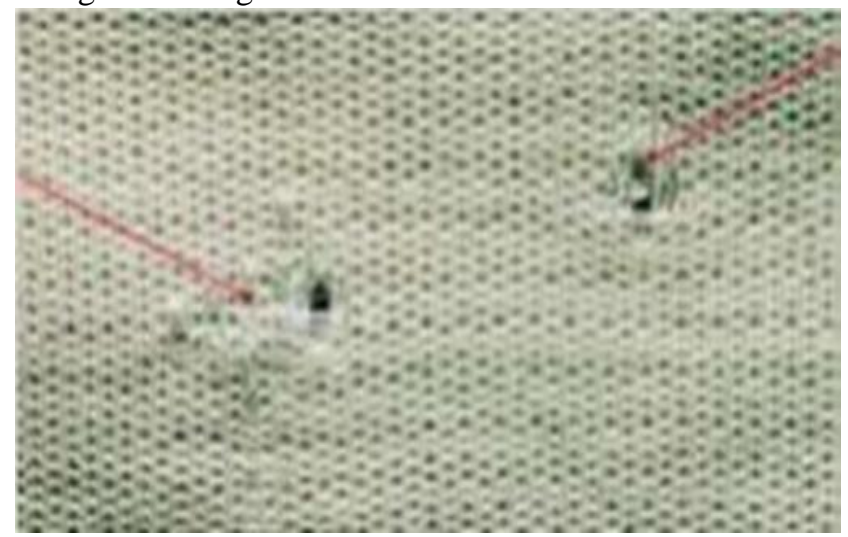

Fig. 1. Defects in Knitted Fabric

Round weaving is one of the quickest and most straightforward techniques $(20$ million fastens every moment) of creating textures. Texture flaws are in charge of

Revised Manuscript Received on 14 August, 2019.

J. Ramprabu, Assistant Professor II, Department of Electrical and Electronics Engineering, Kumaraguru College of Technology, Coimbatore, Tamilnadu, India.(Email: Ramprabu.j.eee@kct.ac.in)

Aswini N, Department of Electrical and Electronics Engineering, Kumaraguru College of Technology, Coimbatore, Tamilnadu, India.(Email: aswini.17mes@kct.ac.in). almost $25 \%$ misfortunes in the creation rate. A mechanized imperfection ID framework .upgrades the item quality and improves profitability brings about both client requests and to diminish the costs related with off-quality. The opportune recognition of texture imperfection could really compare to ever. By and by, examination is done physically by an individual when a texture is created; the texture roll is taken from the round sewing machine, and after that sent to a review system. The best arrangement is naturally review texture, and to urge upkeep laborers to counteract the generation of deformities, consequently improving item quality. The issue has lead to the identification of two primary imperfections in sewed textures, even and vertical shortcomings. While the principal class is primarily connected with the yarn (quality and the executives), the subsequent classification is identified with the sewing components, for example, needles, sinkers, feeders, etc. The answers for these issues are, for the primary kind, a cautious administration of the yarn, and for the subsequent classification, the recognizable proof and expelling of the imperfect components. Thus, to manage these issues, different specific frameworks were created which can distinguish discontinuities in the yarn being bolstered, surrenders in the sewed texture and blames in the sewing components [1].

Fig. 2. Common defects in knitting machine

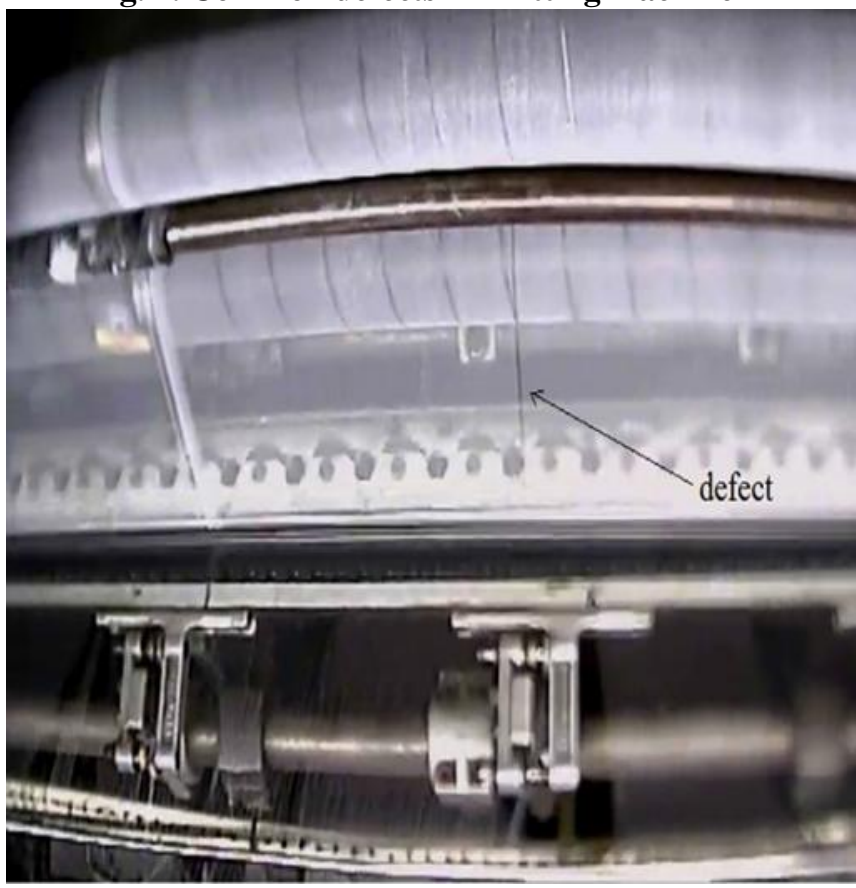

Published By:

Blue Eyes Intelligence Engineering

\& Sciences Publication

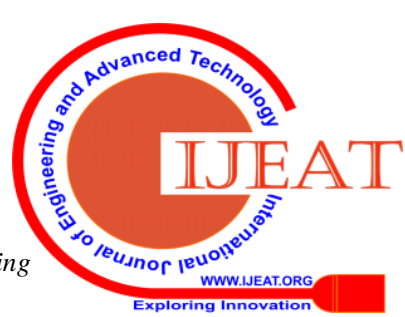


In a prior work, neural system methods were connected for arranging the deficiencies in the roundabout sewing machine. The consequence of the strategy succeeded, yet this system isn't profitable in the business in light of the fact that the entire procedure expends additional time and isn't utilized to decide the deficiency's area [2]. To forestall these issues, in momentum examine, the edge identification procedure was connected to process the picture of roundabout weaved textures. Contingent upon the structure of the weaved texture, imperfections can be arranged into three sorts: vertical, even and territorial deformities. This paper gives a

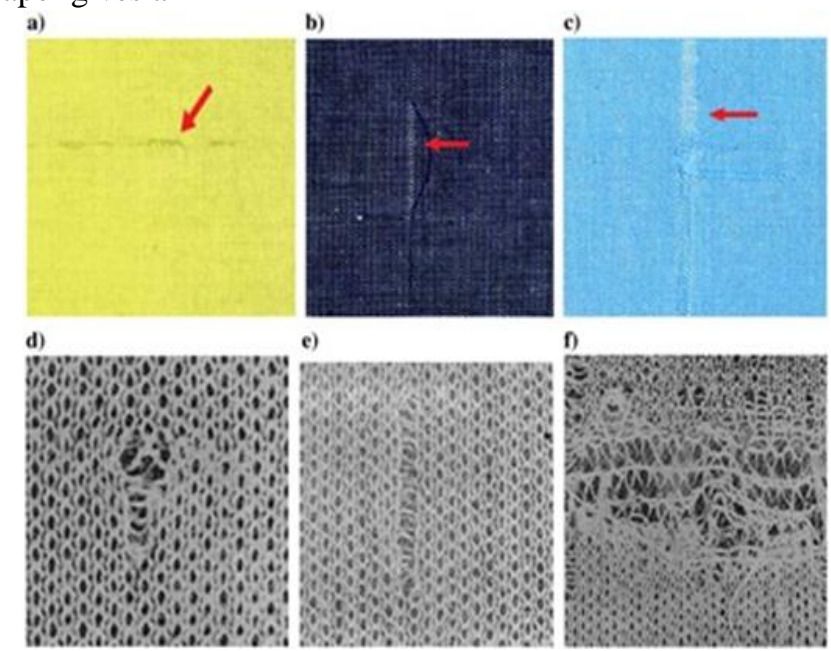

Fig. 3. Various types of defects in fabric

strategy and the framework that created at the weaving machine for the programmed recognition and distinguishing proof of deformities in the sewed textures.

The proposed framework uses picture handling systems to recognize the deformities and decides the area of the imperfection. Furthermore, the primary favorable circumstances of the framework that the stops the machine at whatever point the imperfection was found. The web of things has developed bit by bit. Everything around on the planet is getting associated with the web. Our framework stores the information to the database for the future procedure.

\section{BACKGROUND}

The most recent couple of years have demonstrated some promising patterns in texture deformity discovery inquire about. Notwithstanding, the scientists should a great deal of truly consider deliberate/relative execution investigation bolstered sensible suspicions. The compelling presentation assessment requires cautious choice of informational indexes alongside its reasonable meaning of degree. This will expel any emotional judgment of results and license the clients to get a handle on which calculations are aggressive in which space. Notwithstanding the much advancement inside the most recent decade, the issue of material imperfection location still stays troublesome and needs more consideration. The factual, unearthly and model-based methodologies offer totally various outcomes thus the blend of those methodologies can offer higher outcomes, than it is possible that one independently, and is generally prescribed for future examination [3].

\section{A. Manual Inspection}

Texture review is normally done on a texture test machine. These machines are structured so moves of fabric might be mounted behind the assessment table beneath the sufficient light and rerolled as they leave the table Imperfections might be seen quickly with these machines on the grounds that the assessors incorporate a magnificent read of the texture and in this manner the texture needn't be turned around to sight deserts. The moderate speed of weaving texture is insufficient to remain a human examiner involved and manual investigation is in this manner uneconomical.

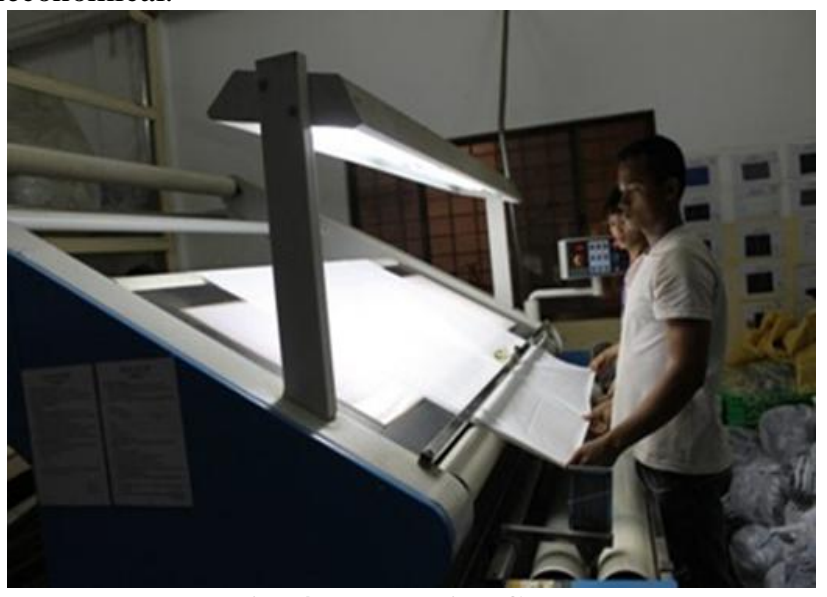

Fig. 4. Four Point Check

[Likewise, the similarly threatening examination area close to the weaving apparatus isn't suitable for human scrutiny. The conventional issue recognizable proof technique is to dispose of the sewn texture moves from the sewing machines and unroll them on the investigation table at a nearly superior speed of 8-20 meters for every moment. At the point when an assessor sees a deformity on the moving texture, he stops the machine, records the imperfection and its area, and begins the machine once more.

\section{A. Automated Inspection}

The mechanization of the visual investigation procedure is a flexible issue and requires complex association among different framework segments. J.L.Rahejahave demonstrated that the interest in the robotized texture assessment framework is financially alluring when a decrease in work force cost and related advantages are considered [5]. The framework comprises of a bank of cameras prearranged in parallel over the net to be filtered, a PC comfort facilitating (single or a variety of) processors, the edge grabber, a lighting framework and furthermore the supporting electrical and mechanical interfaces for the request machine [6]. There are 2 normal assortments of examining procedures used for the fabric review cameras: line filtering and zone checking.

\section{B. Prior Literature Review}

The prior AVI surveys could not focus on the scrutiny of fabric defects, due to their wide coverage on inspection

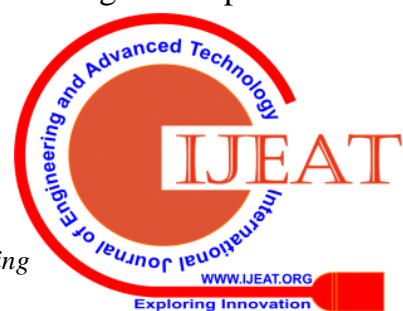


difficulty. Among these only survey in has included a short paragraph which discusses the fabric defect detection techniques in six references. The review presented as excluded fabric inspection mainly due to their coverage on a range of AVI techniques for other manufacturing products. Moreover, there have been more than a few key developments in AVI method for fabric faults in the last 10 years.

In addition, due to the speedily decreasing cost of sensing and computing power, more than a few new algorithms have been proposed for the fabric examination. The fabric flaw exposure approaches are not limited to the images acquired from a digital camera; Sheen et al. have presented an ultrasonic imaging structure for material web scrutiny, illustrates the use of reflected infrared frequencies while the fabric assessment using the knowledge-based a system to trace fabric flaws have been proposed. The literature for cloth defect detection exploitation digital imaging is kind of huge [7].The relevant papers seem in journals and conferences associated with laptop vision, textile, industry applications, and pattern recognition [8].

The algorithms for the defect exposure utilized in some commercially out there systems haven't been according within the prose since of the property constraints. Therefore, the main focus of this paper is on the hypothetical algorithms developed for the fabric review instead of on actual scrutiny systems. On the other hand, only a little fabric inspection systems, although very expensive, are at present existing in the marketplace and a quantity of these are consolidated.

\section{METHODOLOGY USED}

The high speed WebCam is used to capture the images of the knitted fabric [9].

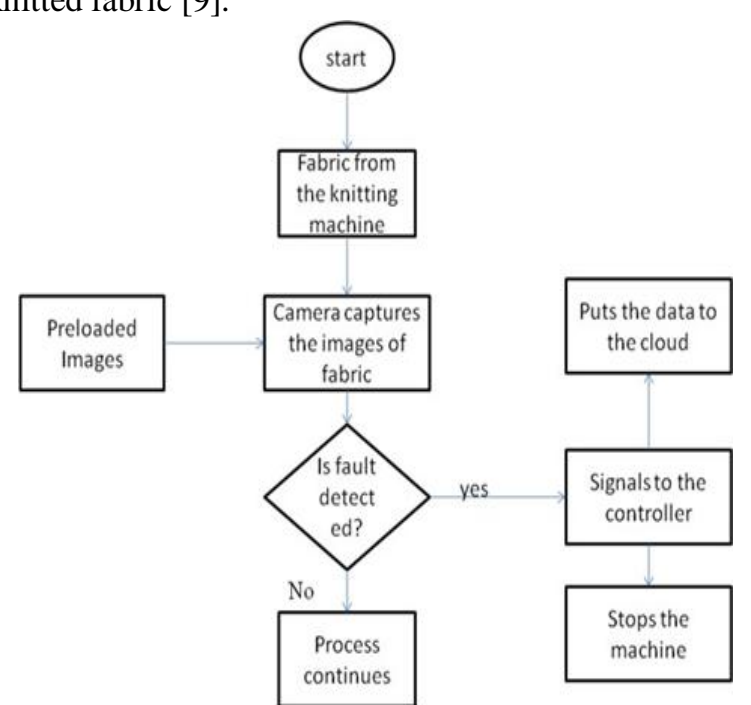

Fig. 5. Flow Chart

\section{A. Image Processing Techniques}

Image preprocessing plays the significant responsibility in it [10].The input image provided to the classification will be the real-time image and will be compared with the record available with the system [11].

The steps involved in this procedure are as follows:-

Capturing Image

Converting RGB to Gray

\section{Histogram}

Histogram Equalization

Conversation of Binary

Detection of Edge

Extraction of Features

Edge Detection Method: the majority of the structure in sequence of an image is bordered in edges. So first we distinguish these edges in an image and by using these filters and then by enhancing those regions of an image which contains edges, the sharpness(high frequency) of the image will amplify and an image will turn out to be clearer [12]. Here are some of the filters for edge detection is

1) Prewitt Operator 2)Sobel Operator

3)Robinson Compass Masks 4)Krisch Compass Masks 5)Laplacian Operator.

Canny edge detection 7)Gabor filter

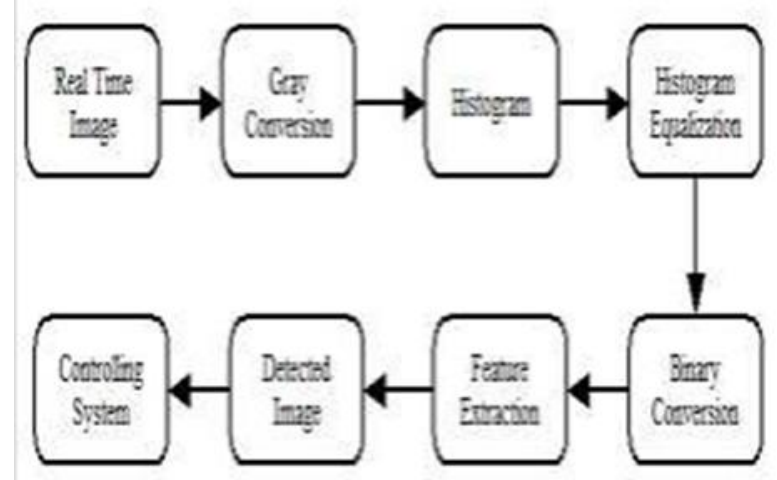

Fig. 6. Steps Involved in Edge Detection

\section{HARDWARE ARCHITECTURE}

The block diagram consists of

Raspberry pi B+

Lora gateway

Motor

WebCamera

Buzzer

Monitor

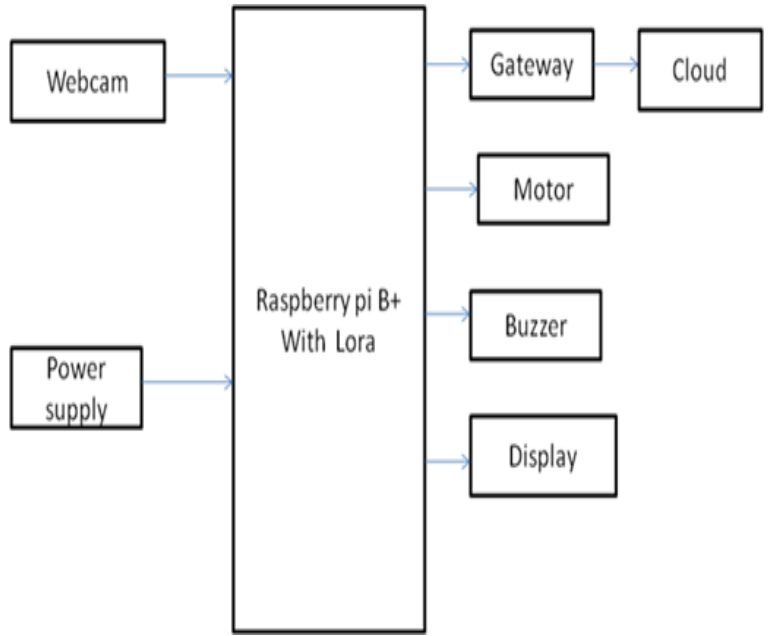

Fig. 7. Block Diagram

Blue Eyes Intelligence Engineering

\& Sciences Publication 


\section{IMAGE PROCESSING BASED PRODUCTION FLAW DETECTION IN KNITTING}

\section{A. Lora Gateway}

LoRa is a fantastic advancement in wireless communication. Lora gateway is used to connect the raspberry pi with the network where the data is stored.

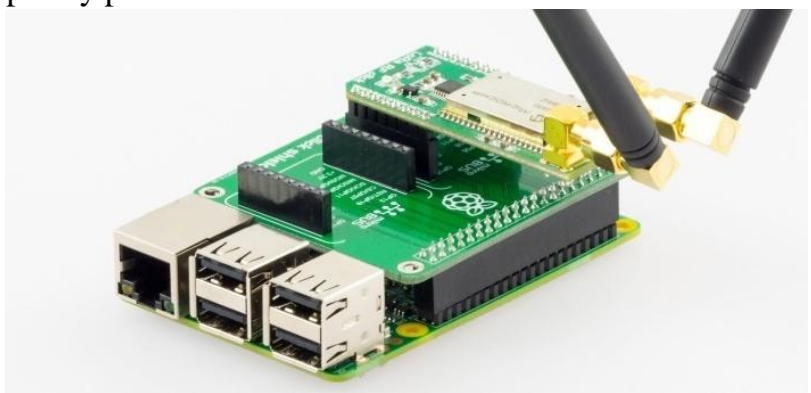

Fig. 8. Raspberry pi B+ with Lora Gateway

\section{HYSTERESIS THRESHOLD}

This stage additionally removes tiny pixels noises on the belief that edges are long lines.

\section{SOFTWARE}

\section{A. Python Programming}

Python-specific IDE and Editors are

1)Pycharm

2)Spyder

3)Thonny

Here, we use spyder tool for the edge detection process.Spyder is an open-source Python IDE that's optimized for knowledge science workflows.Spyder comes enclosed with the anaconda package manager distribution, thus betting on your setup you'll have already got it put in on your machine.

\section{B. OpenCV}

It is a prominent multistage calculation created by John F. canny.

\section{Noise Reduction}

Initial step is to dispose of the clamor inside the picture with a $5 \times 5$ Gaussian channel.

\section{Finding Intensity Gradient :}

At that point the smoothed picture is sifted with a Sobel bit in both level and vertical heading to get the principal subsidiary in a flat bearing (Gx) and vertical course(Gy). Gradient direction is always per?pendicular to edges. Edge_Gradient $(\mathrm{G})=\mathrm{G} 2 \mathrm{x}+\mathrm{G} 2 \mathrm{y}$

$$
\text { Angle }(?)=\tan ? 1(\mathrm{GyGx})
$$

\section{Non-maximum Suppression}

After a force angle established, a full output of the picture is finished. It expels any undesirable pixels which may not comprise the edge. For this, at each pixel, the pixel is checked on the off chance that it is a nearby most extreme in its neighborhood toward the angle. Check the picture underneath:

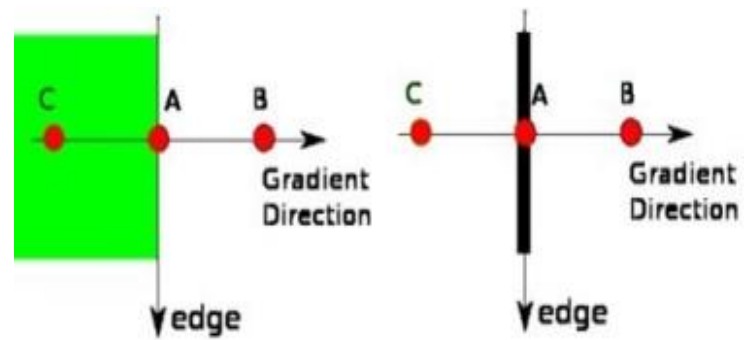

Fig. 9. Image

The result will get is a binary image with thin edges.

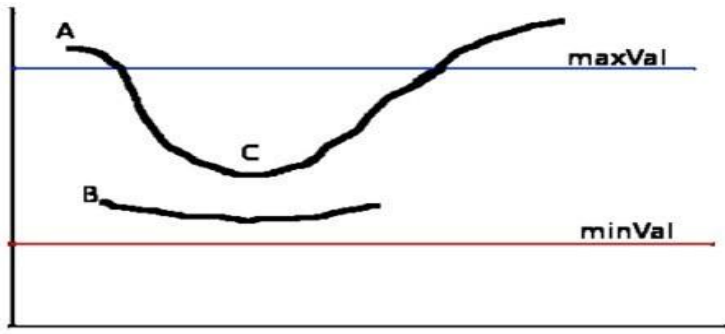

Fig.10. Image

\section{Hysteresis Threshold}

This stage additionally removes tiny pixels noises on the belief that edges are long lines.

C. The Things Network

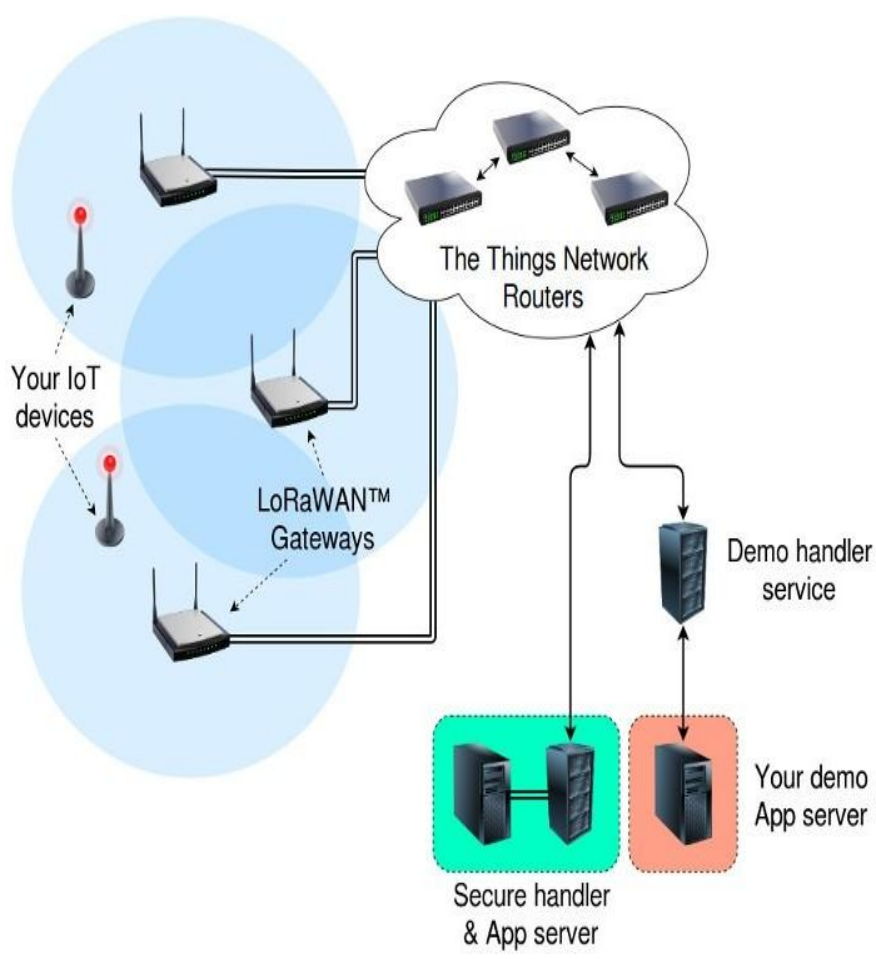

Fig. 11.Iot platform 


\section{EDGE DETECTION RESULT}

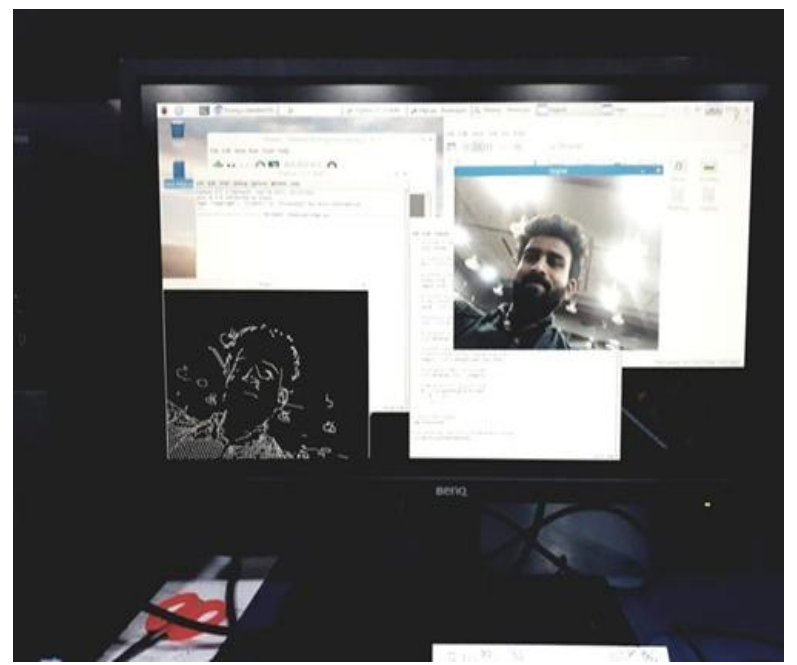

Fig. 12. Edge Detection Result

\section{RESULT}

The result of the proposed was shown below

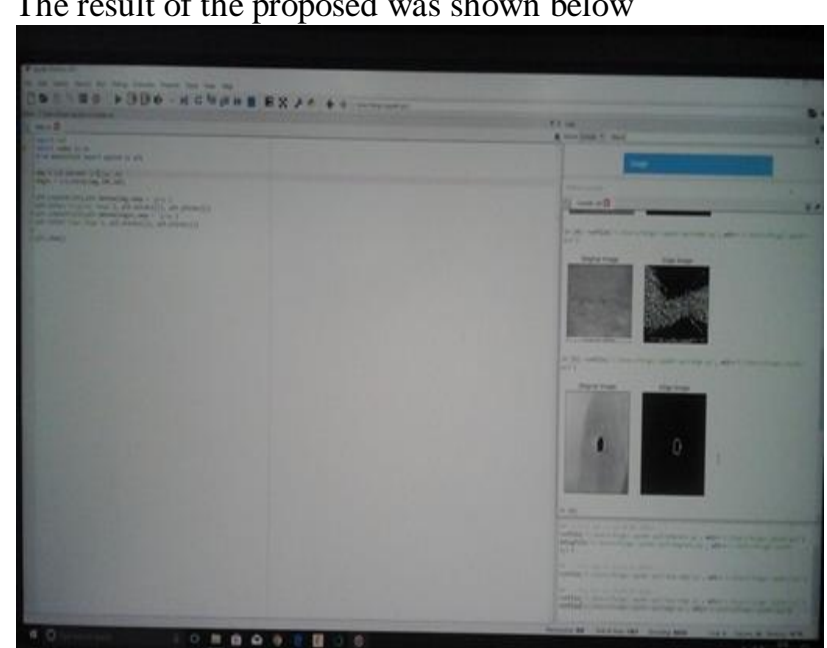

Fig. 13.OpenCV Result

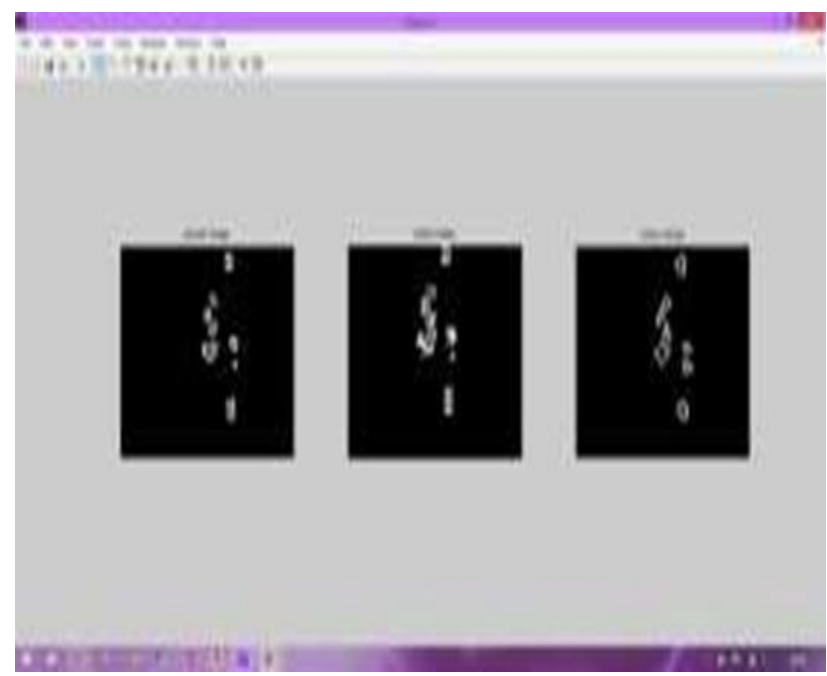

Fig. 14. Defect detection and identification of the location

\section{MERITS}

- $\quad$ Retrofit table device

- $\quad$ Applicable to all colors, patterns and materials
- $\quad$ Reduction in production downtime and imperfect goods

- $\quad$ Time and cost savings due routine adjustment

- Distinguishes between indicator faults and holes

- $\quad$ Also detects faults on high-speed equipment and all types of knitting apparatus.

\section{CONCLUSION}

This undertaking gave a framework built up the reason for checking and identifying issues during the creation of texture in round about sewing machines. The System can be collected in any round machine and uses picture preparing as a way to deal with recognize blames and screen the sewing procedure. It supplies the professional with a critical arrangement of data concerning the real parameters of sewing creation. An outline of the framework was displayed, with specific accentuation on the techniques utilized for recognizing the flaws their favorable circumstances and disadvantages. The arrangement of apparatuses introduced are able to do effectively recognize and find blames in on line creation, therefore adding to the decrease of stop and fix times, and hence lessening flawed knitwear.

The $88 \%$ of production losses were reduced by the proposed system. And also the time consumption during defect detection and correction was minimized.

\section{FURTHER DEVELOPMENTS}

Further examination is being performed to improve the systems of shortcoming discovery. A database is being gathered so as to continue with the following phase of this application, which is the advancement of an example acknowledgment framework for recognizable proof of the shortcomings. A few examinations were at that point made utilizing LabVIEW systems with promising outcomes. It is additionally proposed to stretch out this application to other weaving structures and machines.

\section{REFERENCES}

1. H. Y. T. Ngan, "Pang GKH, Yung NHC," Image Vis Comput, vol. 7, no. 442-458, 2011.

2. P. Y. Kumbhar and R. N. TejaswiniMathpati, "'Textile Fabric Defects Detection and Sorting Using Image," vol. 3, pp. 2016-2349.

3. "Thilepa R.: A Paper on Automatic Fabrics Fault Processing using," vol. 1, pp. 88-99, 2010.

4. Y. S. H. r. Long, "Adaptive detection of weft-knitted fabric defects based on machine vision system,", Journal of the Textile Institute, vol. 102, no. 10, pp. 823-836, 2011.

5. J. L. Raheja, B. Ajay, and A. Chaudhary, "Real time fabric defect detection system on an embedded DSP platform, Opt," Int. J. Light Electron. Opt, vol. 124, pp. 21-2013.

6. H. Y. T. Ngan, G. K. H. Pang, and N. H. C. Yung, "Automated fabric defect detection-a review," Image Vision Comput, vol. 29, pp. 442-458, 2011.

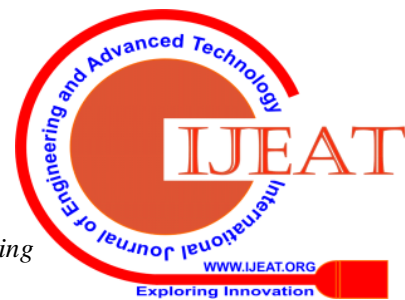


7. Nisi, K., B. Nagaraj, and A. Agalya. "Tuning of a PID controller using evolutionary multi objective optimization methodologies and application to the pulp and paper industry." International Journal of Machine Learning and Cybernetics (2018): 1-11.

8. C. F. J. Kuo, C. Y. Shih, C. C. Huang, and Y.-M. Wen, "Image inspection of knitted fabric defects using wavelet packets,,"Textile Research Journal, 2015.

9. T. Pham, "Optimization of texture feature extraction algorithm," 2010.

10. "camera," 2016. [Online]. Available: http://www.baslerweb.com/en/ products/cameras/linescan-cameras

11. B. Venkatesan, U. S. Ragupathy, P. Vidhyalakshmi, and B., Vinoth, Inspection of faults in textile web materials using wavelets and, 2012.

12. "B.Karunamoorthy,Dr.D.Somasundareswari,S.P.Sethu

"AUTOMATED PATTERNED FABRIC FAULT DETECTION USING IMAGE PROCESSING TECHNIQUE INMATLAB".InternationalJournal of," vol. 4, 12015.

13. P. Y. Kumbhar, T. Mathpati, R. Kamaraddi, and N. Kshirsagar, "Textile fabric defects detection and sorting using image processing, Int," J. Res. Emerging Sci.Technol, vol. 3, pp. 19-24, 2016. 